\title{
Parallel MRI Noise Correction: An Extension of the LMMSE to Non Central $\chi$ Distributions
}

\author{
Véronique Brion $^{1,2}$, Cyril Poupon ${ }^{1,2}$, Olivier Riff ${ }^{1,2}$, Santiago Aja-Fernández ${ }^{3}$, \\ Antonio Tristán-Vega ${ }^{3}$, Jean-François Mangin ${ }^{1,2}$, Denis Le Bihan ${ }^{1,2}$, \\ and Fabrice Poupon ${ }^{1,2}$ \\ 1 CEA I2BM NeuroSpin, Gif-sur-Yvette, France \\ 2 IFR 49, Paris, France \\ 3 LPI, Universidad de Valladolid, Spain
}

\begin{abstract}
Parallel MRI leads to magnitude data corrupted by noise described in most cases as following a Rician or a non central $\chi$ distribution. And yet, very few correction methods perform a non central $\chi$ noise removal. However, this correction step, adapted to the correct noise model, is of very much importance, especially when working with Diffusion Weighted MR data yielding a low SNR. We propose an extended Linear Minimum Mean Square Error estimator (LMMSE), which is adapted to deal with non central $\chi$ distributions. We demonstrate on simulated and real data that the extended LMMSE outperforms the original LMMSE on images corrupted by a non central $\chi$ noise.
\end{abstract}

\section{Introduction}

As many other imaging techniques, Magnetic Resonance Imaging (MRI) is very sensitive to noise in the data. Noise decreases image quality in MRI and particularly in diffusion weighted MRI (dMRI) where the diffusion indicator in the tissues is the measured signal loss. Moreover, noise has higher corrupting effects when using high b-values.

The MR signal is complex with two real and imaginary channels each corrupted by a zero-mean uncorrelated Gaussian noise [1]. It is most common to work on the magnitude of the measured signal in order to avoid the artefacts due to the phase. Because of the non linear square root function used to get it, the measured magnitude will not follow a Gaussian, but a more complex distribution. In case of a multiple-channel acquisition, with a Sum-of-squares (SoS) reconstruction, the noisy magnitude follows a non-central $\chi$ (nc- $\chi$ ) distribution 2. In the case of Generalized Autocalibrating Partially Parallel Acquisition (GRAPPA) reconstruction, [3] reminds that the noise is non-stationnary, so in this case, the nc- $\chi$ hypothesis becomes a good approximation. A particular case of the nc- $\chi$ distribution, namely the Rician distribution, appears in case of a single-channel acquisition, or when using the Sensitivity Encoding for Fast MRI (SENSE) algorithm [4]. For high signal to noise ratios (SNRs), a nc- $\chi$ distribution can be approximated by a Gaussian distribution, and least square estimators can be used efficiently. At high b-values however, the SNR drops and 
the signal depicts a systematic bias. Least square estimators cannot be used anymore because the mean operator does not converge to the noise-free magnitude. This makes the nc- $\chi$ process more difficult to correct than a Gaussian one. High Angular Resolution Diffusion Imaging (HARDI) or HYbrid Diffusion Imaging (HYDI) are usually performed at those high b-values yielding low SNR data on clinical systems. That is why a correction taking into account the nc- $\chi$ process is essential to improve the data quality and analysis.

Numerous techniques, adapted to a Rician noise, were proposed to correct MR data. A review of many of them can be found in [5]. But, most of these methods are not adapted to correct a nc- $\chi$ noise. To our knowledge, only two techniques deal with this more complex process. The first one, the analytically exact correction scheme developed in [6] relies on a fixed point formula of SNR and a correction factor to extract the noise-free signal. The second one is based on a framework which transforms the magnitude signals so that they follow Gaussian distributions, in order to use the least square approach on them [7.

In this paper, we propose to extend the method proposed by Aja-Fernández et al in [5] to the correction of nc- $\chi$ noise. The original technique relies on a Linear Minimum Mean Square Error estimator (LMMSE) that calculates the noise-corrected signals thanks to local estimations of mean and variance. As the LMMSE corrects the magnitude signals directly, any diffusion model can be applied on the corrected diffusion weighted (DW) data, making the method generic. Moreover, the algorithm is easy and fast to compute. Because of all these reasons, the LMMSE was used in many studies ([8, [9], 10, [1] ). That is why an adaptation of the LMMSE to the nc- $\chi$ noise model might be of great interest for all works dealing with Parallel MRI data corrupted by a nc- $\chi$ noise and where the LMMSE is involved. This implies that the noise estimation must also be adapted, as done in [12. First, we explain the extension of the LMMSE to the nc- $\chi$ distribution. Then, we describe the methods to infer the noise standard deviation. Finally, we validate the technique on simulated and real data, applying it to the popular Diffusion Tensor (DTI) and analytical Q-ball models.

\section{Methods}

\subsection{Extended LMMSE Model}

The LMMSE of [5] relies on a simple expression of the squared noise-free magnitude:

$$
\hat{S}^{2}=E\left(S^{2}\right)+\operatorname{Cov}\left(S^{2}, M^{2}\right) \operatorname{Var}\left(M^{2}\right)^{-1} \times\left(M^{2}-E\left(M^{2}\right)\right),
$$

with $S^{2}$ being the squared noise-free magnitude, $\hat{S}^{2}$ being its estimation and $M$ being the measured magnitude. This expression is true for any voxel and any diffusion orientation, and to simplify we do not add their corresponding values. $\operatorname{Cov}\left(S^{2}, M^{2}\right)$ is the covariance of $S^{2}$ and $M^{2}$ and $\operatorname{Var}\left(M^{2}\right)$ is the variance of $M^{2}$. (11) allows to estimate the noise-free magnitude and relies on the knowledge of $E\left(S^{2}\right), \operatorname{Cov}\left(S^{2}, M^{2}\right)$ and $\operatorname{Var}\left(M^{2}\right)$. Their expressions for a Rician noise are 
given in [5. We propose to follow the same process with the assumption of a multiple-coil acquisition yielding a nc- $\chi$ noise. The nc- $\chi$ distribution is given by:

$$
P(M ; S, \sigma, n)=\frac{M}{\sigma^{2}}\left(\frac{M}{S}\right)^{n-1} \exp \left(-\frac{M^{2}+S^{2}}{2 \sigma^{2}}\right) \cdot I_{n-1}\left(\frac{S M}{\sigma^{2}}\right),
$$

with $\sigma$ being the noise standard deviation of the Gaussian noises present on the complex acquisition channels, $n$ being the number of channels used in the acquisition and $I_{n-1}$ being the $(n-1)$ th order modified Bessel function of the first kind. In such multiple-channel acquisitions, the measure of the magnitude of the magnetization is generally performed using an $\mathrm{n}$ element phased array antenna. First, we consider the commonly used "Sum-of-Squares" (SoS) reconstruction method to get $M=\left(\sum_{k=1}^{n}\left(S_{r_{k}}+B_{r_{k}}\right)^{2}+\left(S_{i_{k}}+B_{i_{k}}\right)^{2}\right)^{1 / 2}$, where $S=\left(\sum_{k=1}^{n} S_{r_{k}}^{2}+S_{i_{k}}^{2}\right)^{1 / 2}=\left(\sum_{k=1}^{n} S_{k}^{2}\right)^{1 / 2} . S_{r_{k}}$ and $S_{i_{k}}$ are the real and imaginary parts of the noise-free signal $S_{k}$ received by the coil $k ; B_{r_{k}}$ and $B_{i_{k}}$ are the real and imaginary parts of the noise corrupting the signal $S_{k}$. They are supposed to be zero-mean, uncorrelated and independant Gaussian noises. Thanks to these assumptions, the variance and covariance terms can be written:

$$
\left\{\begin{array}{l}
\operatorname{Var}\left(M^{2}\right)=E\left(M^{4}\right)-E\left(M^{2}\right)^{2}, \\
\operatorname{Cov}\left(S^{2}, M^{2}\right)=E\left(S^{4}\right)+2 n \sigma^{2} E\left(S^{2}\right)-E\left(S^{2}\right) E\left(M^{2}\right) .
\end{array}\right.
$$

Injecting both variance and covariance expressions in the equation (11), we obtain:

$$
\hat{S}^{2}=E\left(S^{2}\right)+\frac{\left(E\left(S^{4}\right)+2 n \sigma^{2} E\left[S^{2}\right]-E\left(S^{2}\right) E\left(M^{2}\right)\right)}{\left(E\left(M^{4}\right)-E\left(M^{2}\right)^{2}\right)} \times\left(M^{2}-E\left(M^{2}\right)\right)
$$

Now, if we use the 2 nd and 4 th order moments of the nc- $\chi$ distribution, we have:

$$
\left\{\begin{array}{l}
E\left(S^{2}\right)=E\left(M^{2}\right)-2 n \sigma^{2} \\
E\left(S^{4}\right)=E\left(M^{4}\right)-4(n+1) \sigma^{2} E\left(M^{2}\right)+4 n(n+1) \sigma^{4},
\end{array}\right.
$$

and the LMMSE equation (4) for a nc- $\chi$ noise can finally be expressed as:

$$
\hat{S}^{2}=\left\langle M^{2}\right\rangle-2 n \sigma^{2}+\left(1-\frac{4 \sigma^{2}\left[\left\langle M^{2}\right\rangle-n \sigma^{2}\right]}{\left\langle M^{4}\right\rangle-\left\langle M^{2}\right\rangle^{2}}\right) \times\left(M^{2}-\left\langle M^{2}\right\rangle\right)
$$

As done in [5], under the assumption of local ergodicity, we replaced the expectation $E(\cdot)$ by $\langle\cdot\rangle$, a local spatial mean calculated on a neighborhood. For a single channel acquisition (i.e. $n=1$ ), (6) simplifies into its Rician form, as expected.

Practically, employing equation (6) only requires a good estimate of the noise standard deviation $\sigma$. Obtaining such a good estimate is challenging and we propose to address this point in the following subsection.

\subsection{Noise Standard Deviation Estimation}

In this subsection, 2 methods are introduced to infer $\sigma$; a first one relying on the analysis of the signal present in the background of the data; and a second one that does not depend on the presence of a background in the data. 
With the Necessity of a Background. $\sigma$ can easily be estimated using the information provided by the background of the image. The method proposed by Aja-Fernández et al [12, which does not necessarily need a background segmentation, produces the estimate $\hat{\sigma}_{A F}$ of $\sigma$ such that:

$$
\hat{\sigma}_{A F}=\left(\sqrt{2}(n)_{\frac{1}{2}}\right)^{-1} \operatorname{mode}\left(\left\langle M_{b g}(\mathbf{v})\right\rangle\right)
$$

where $M_{b g}(\mathbf{v})$ is the measured magnitude at the voxel $\mathbf{v}$ in the background region; mode $\left(\left\langle M_{b g}\right\rangle\right)$ is the distribution mode of the mean of $M_{b g}(\mathbf{v}) ;(x)_{n}$ is the Pochhammer symbol defined as $(x)_{n}=\frac{\Gamma(x+1)}{\Gamma(x-n+1)}$ with $\Gamma$ the Gamma function.

Without the Necessity of a Background. An interesting method to estimate $\sigma$ without background knowledge was developed by Rajan et al [13], but for Rician noise only. We have extended it to nc- $\chi$ noise. The technique requires to estimate the variance $\sigma_{M}^{2}$ and the skewness $\gamma$, at each voxel of the image, using:

$$
\begin{gathered}
\sigma_{M}^{2}=E\left(M^{2}\right)-E(M)^{2} \\
\gamma=\left(2 E(M)^{3}-3 E(M) E\left(M^{2}\right)+E\left(M^{3}\right)\right) / \sigma_{M}^{3},
\end{gathered}
$$

where the expectations $E(\cdot)$ can also be replaced by a local spatial mean $\langle\cdot\rangle$. The method relies on the computation of a local correction factor $\varphi$ which tunes the proximity of the nc- $\chi$ distribution toward the central $\chi$ distribution for very low SNRs, or toward the Gaussian distribution for very high SNRs. First, a lookup table $\varphi(\gamma)$ is created between the local correction factor $\varphi$ and the local skewness $\gamma$ for a nc- $\chi$ distribution with a given $n$, by varying the value of $S$ and keeping $\sigma$ constant. In order to build this lookup table, the 3 first nc- $\chi$ moments have been calculated from the range of values of $S$ and $\sigma$ :

$$
\begin{aligned}
& E(M)=\sqrt{2} \sigma(n)_{\frac{1}{2}} \times{ }_{1} F_{1}\left(-\frac{1}{2}, n,-\frac{S^{2}}{2 \sigma^{2}}\right) \\
& E\left(M^{2}\right)=2 n \sigma^{2}+S^{2} \\
& E\left(M^{3}\right)=\frac{2}{3} \sqrt{2} \sigma^{3}(n)_{\frac{1}{2}} \times{ }_{1} F_{1}\left(-\frac{3}{2}, n,-\frac{S^{2}}{2 \sigma^{2}}\right),
\end{aligned}
$$

with ${ }_{1} F_{1}(a, b, c)$ being the confluent hypergeometric function. Then, $\gamma$ is obtained by injecting the 3 expressions above in (9). In the same manner, the local variance $\sigma_{M}$ is computed using (8). Finally, $\varphi$ is calculated from $\varphi=\sigma^{2} / \sigma_{M}^{2}$. Once the $\varphi(\gamma)$ lookup table is computed and starting from the local estimates $\sigma_{M}^{2}$ (8) and $\gamma(9)$, the final noise standard deviation can be estimated from:

$$
\hat{\sigma}_{R}=\sqrt{\operatorname{mode}\left(\varphi \times \sigma_{M}^{2}\right)},
$$

where mode $\left(\varphi \times \sigma_{M}^{2}\right)$ is the distribution mode of $\varphi \times \sigma_{M}^{2}$ calculated on the image. 


\section{Results and Discussion}

We used our extended LMMSE method on both simulated and real DW data perturbed by a nc- $\chi$ noise, and we compared it to the original LMMSE method adapted to a Rician noise.

\subsection{Standard Deviation Estimations}

First, we tested the estimation methods described in section 2.2 on a simulated T1 data taken from the BrainWeb database [14]. The original image is considered as the ground truth. We added a high nc- $\chi$ noise using $n=4$ channels that correspond to the standard case of the 12 channel head coil antenna available on TimTrio systems in which the 12 channels are coupled in 4 associations of 3 channels, and we used a standard deviation $\sigma=70.0$ leading to a very unfavorable situation. We made the same experiment on a synthetic DW field depicting crossing fiber bundles simulated using a Gaussian mixture model with nc- $\chi$ noise addition $(n=4, \sigma=30.0)$. Table 1 shows that very good estimations can be found by the first and second methods, with the knowledge of the background-signal. Without it, the second method still gives good results.

Table 1. Different $\sigma$ estimations. $\hat{\sigma}_{A F}$ is obtained with the method of [12] using the background (bg) of the image. $\hat{\sigma}_{R}^{w / b g}$ and $\hat{\sigma}_{R}^{w o / b g}$ are obtained with the method using equation (10), performed respectively with and without the bg.

\begin{tabular}{|c|c|c|c|}
\hline Simulated data & Ground truth $\sigma$ & $\mathrm{w} / \mathrm{bg}$ necessity & wo/bg necessity \\
\hline T1 image & 70.0 & $\hat{\sigma}_{A F}=70.0$ & $\hat{\sigma}_{R}^{w / b g}=70.1$ and $\hat{\sigma}_{R}^{w o / b g}=73.4$ \\
\hline DW field & 30.0 & $\hat{\sigma}_{A F}=29.5$ & $\hat{\sigma}_{R}^{w / b g}=30.0$ and $\hat{\sigma}_{R}^{w o / b g}=30.9$ \\
\hline
\end{tabular}

\subsection{Simulated Data}

We used both simulated data already mentioned in section 3.1 . Using a $5 \times 5 \times 5$ neighborhood, we tested both versions of the LMMSE: the LMMSE correction introduced in [5], adapted for a Rician noise (Rice LMMSE) and the extended LMMSE correction introduced in this work following equation (6), adapted for a nc- $\chi$ noise (nc- $\chi$ LMMSE). Before the computation of the LMMSEs, $\sigma$ was estimated using (7), with $n=1$ for the Rice LMMSE and with $n=4$ for the nc- $\chi$ LMMSE. Concerning the DW field, the Orientation Distribution Functions (ODFs) were processed using the analytical Q-ball model [15, with the maximum spherical harmonic $(\mathrm{SH})$ order $N=8$ and the Laplace-Beltrami regularization factor $\lambda=0.006$. In comparison to the Rice LMMSE, Fig. 1 shows that the nc- $\chi$ LMMSE presents much better performance: good noise cleaning and low smoothing effects. This reveals the importance of taking the correct noise model into account in the LMMSE tool. To quantitatively validate this result, we measured the quadratic errors:

$$
\epsilon=\sum_{\mathbf{v}=1}^{M}\left(\sum_{j=1}^{N}\left(\mathbf{C}_{\mathbf{v}}^{\mathbf{D W I}}(j)-\tilde{\mathbf{C}}_{\mathbf{v}}^{\mathbf{D W I}}(j)\right)^{2}\right)
$$




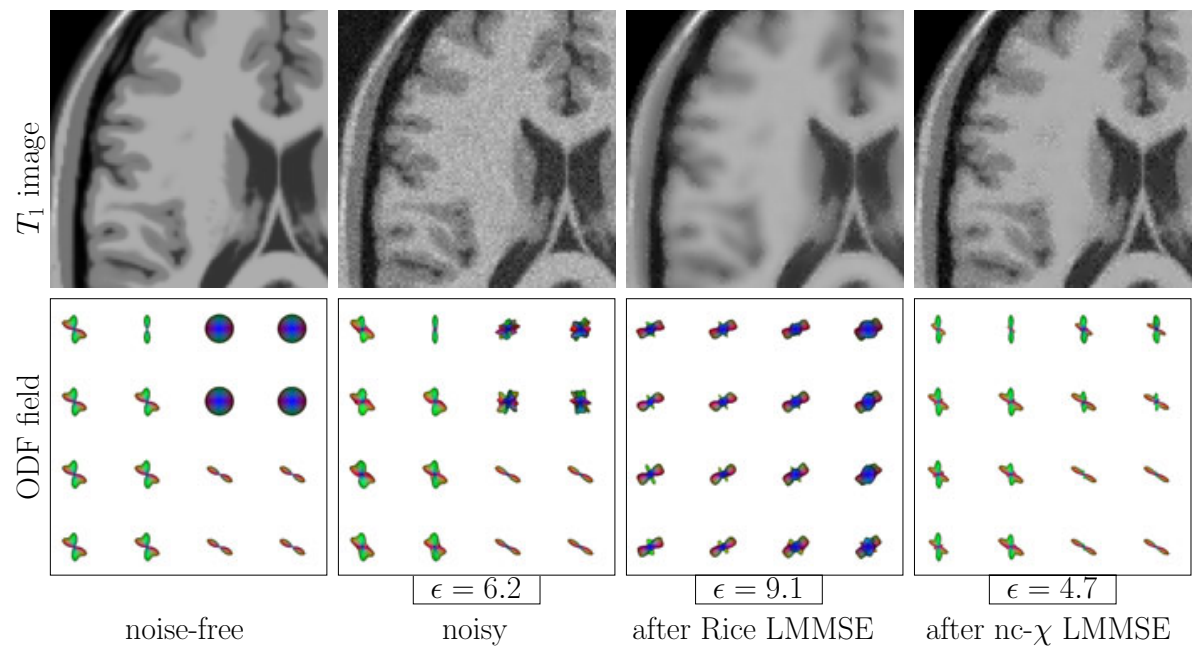

Fig. 1. Comparison between both LMMSEs on simulations of a T1 image and of ODFs

where $\mathbf{C}_{\mathbf{v}}^{\mathbf{D W I}}$ is the $\mathrm{SH}$ coefficient vector of the noise-free DW signal of voxel $\mathbf{v}$ ( $M$ is the number of voxels in the field) and $\tilde{\mathbf{C}}_{\mathbf{v}}^{\mathbf{D W I}}$ is either the noisy or the corrected coefficient vector. Whereas $\epsilon$ increased after the Rice LMMSE, it dramatically decreased after the nc- $\chi$ LMMSE.

\subsection{Real DW Data}

DW data were collected on a Magnetom Tim Trio 3T MRI system (Siemens Medical Solutions, Erlangen, Germany), using a gradient sampling scheme of 60 orientations uniformly distributed over a sphere at $b=4500 \mathrm{~s} . \mathrm{mm}^{-2}$. A further reference T2 weighted volume was acquired at $b=0 s . \mathrm{mm}^{-2}$. The acquisition parameters were as follows: $T_{E} / T_{R}=116 \mathrm{~ms} / 14 \mathrm{~s}$, field of view $F O V=198 \mathrm{~mm}$, matrix $128 \times 128$, resolution $1.7 \times 1.7 \times 1.7 \mathrm{~mm}^{3}$, GRAPPA factor of 2 , read bandwidth $R B W=1628 \mathrm{~Hz} /$ pixel and a number of coils of $12 \mathrm{in}$ an architecture of $4 \times 3$ coupled coils, so $n=4$ in this case. We tested our method on the DTI model (color-encoded (RGB) map) and on the analyticall Q-ball model (Generalized Fractional Anisotropy (GFA) and ODFs) with $N=8$ and $\lambda=0.006$. Fig. 2 shows the results obtained for the Rice LMMSE and the nc- $\chi$ LMMSE. As for the simulated data, the GFA and RGB maps emphasize the importance of using the nc- $\chi$ noise model, yielding a preservation of the fine details and a limited smoothing effect. On the ODF maps, the Rice LMMSE seems to provide better results, but this is only due to a visual perception of a better coherence induced by an oversmoothing effect. Concerning the ODFs in the red circle, the Rice LMMSE completely overlooks the raw information in contrary to the nc- $\chi$ LMMSE. The latter has a better behavior since it corrects the ODFs from the influence of noise while keeping the right information. 

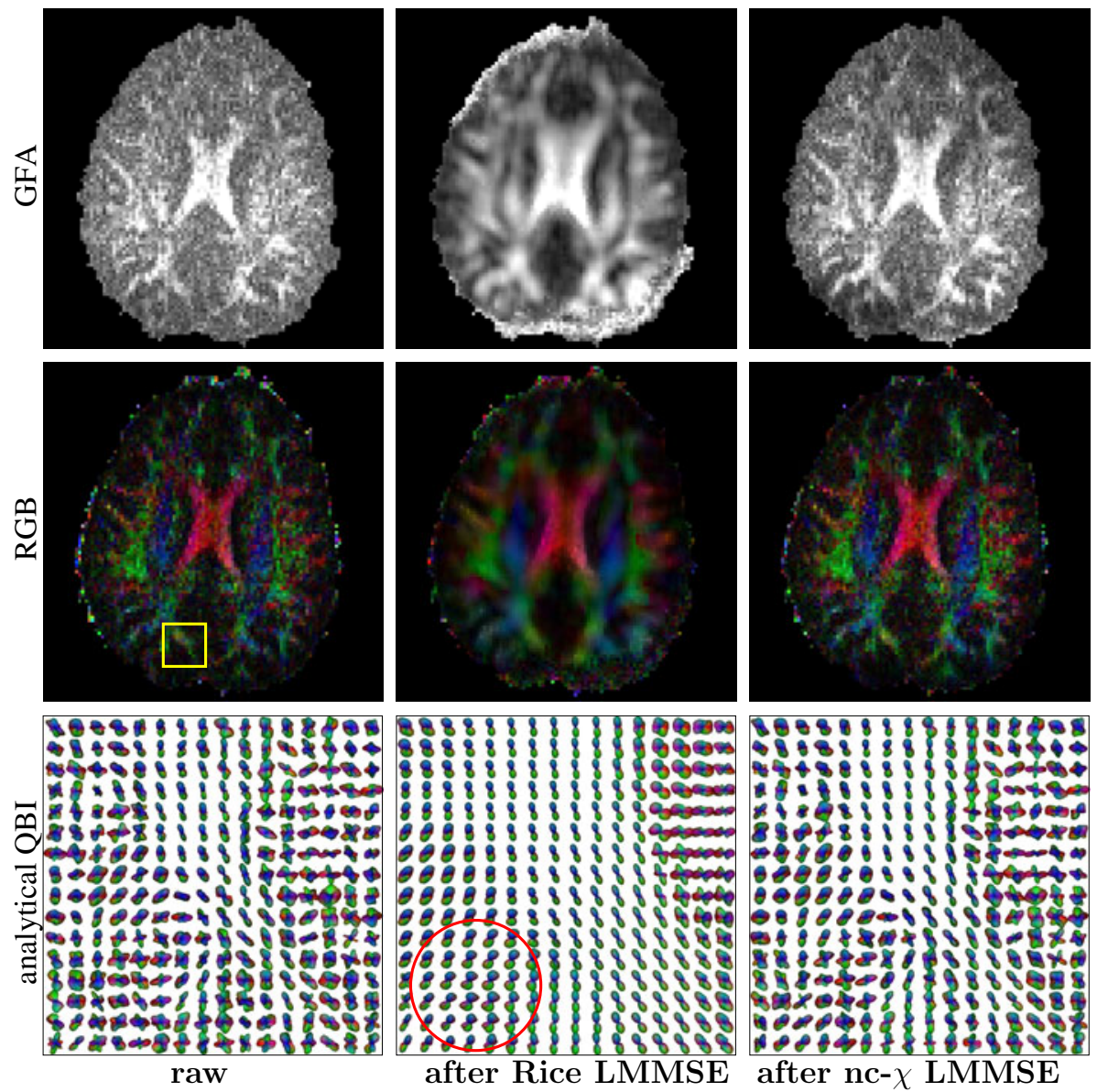

Fig. 2. Comparison between both LMMSEs on GFA, RGB and ODF maps. The ODFs are computed in the yellow ROI shown on the RGB map.

\section{Conclusion}

We have proposed an extension of the LMMSE to nc- $\chi$ noise correction. The comparison between the Rice and the nc- $\chi$ LMMSEs on synthetic and real data successfully assesses the choice of the nc- $\chi$ LMMSE in case of a multiple-coil acquisition yielding a nc- $\chi$ noise with $n>1$. Whereas the Rice LMMSE oversmoothes the images, the nc- $\chi$ LMMSE performs a clearly visible correction with respect to the details, even in very defavorable conditions as at $b=4500 \mathrm{~s} . \mathrm{mm}^{-2}$. The real data we used were obtained with GRAPPA reconstruction, and yet, it has been recently discussed in [3] that an effective number of channels, as well as an effective variance of noise, must be calculated to get a nc- $\chi$ noise model that better fits the data. Both effective calculated parameters are not stationnary and should be evaluated at each voxel of the image. Future work will be done 
to apply this effective parameters' calculations to the nc- $\chi$ LMMSE correction. Last, our method can easily be extended to its recursive form as it was done in [5] for the Rice LMMSE, but this was beyond the scope of this work.

Acknowledgements. The authors thank Mr. Rajan for discussions about the noise estimation method developed in [13] and acknowledge the CONNECT grant and MCIN for grant TEC2010-17982.

\section{References}

1. Henkelman, R.M.: Measurement of signal intensities in the presence of noise in MR images. Med. Phys. 12, 232-233 (1985)

2. Constantinides, C.D., Atalar, E., McVeigh, E.R.: Signal-to-Noise Measurements in Magnitude Images from NMR Phased Arrays. Magn. Reson. Med. 38, 852-857 (1997)

3. Aja-Fernández, S., Tristán-Vega, A., Hoge, W.S.: Statistical noise analysis in GRAPPA using a parametrized noncentral chi approximation model. Magn. Reson. Med. 65, 1195-1206 (2011)

4. Dietrich, O., Raya, J.G., Reeder, S.B., Ingrisch, M., Reiser, M.F., Schoenberg, S.O.: Influence of multichannel combination, parallel imaging and other reconstruction techniques on MRI noise characteristics. MRI 26, 754-762 (2008)

5. Aja-Fernández, S., Niethammer, M., Kubicki, M., Shenton, M.E., Westin, C.F.: Restoration of DWI Data Using a Rician LMMSE Estimator. IEEE Trans. Med. Imag. 27, 1389-1403 (2008)

6. Koay, C.G., Basser, P.J.: Analytically exact correction scheme for signal extraction from noisy magnitude MR signals. J. Magn. Reson. 179, 317-322 (2006)

7. Koay, C.G., Özarslan, E., Basser, P.J.: A signal transformational framework for breaking the noise floor and its applications in MRI. J. Magn. Reson. 197, 108-119 (2009)

8. Krissian, K., Aja-Fernández, S.: Noise-driven anisotropic diffusion filtering of MRI. IEEE Trans. Image Process. 18, 2265-2274 (2009)

9. Caan, M.W.A., Khedoe, G., Poot, D., den Dekker, A., Olabarriaga, S., Grimbergen, K., van Vliet, L., Vos, F.: Adaptive noise filtering for accurate and precise diffusion estimation in fiber crossings. In: Jiang, T., Navab, N., Pluim, J.P.W., Viergever, M.A. (eds.) MICCAI 2010. LNCS, vol. 6361, pp. 167-174. Springer, Heidelberg (2010)

10. Tristán-Vega, A., Aja-Fernández, S.: DWI filtering using joint information for DTI and HARDI. Med. Image Anal. 14, 205-218 (2010)

11. Brion, V., Kezele, I., Riff, O., Descoteaux, M., Mangin, J.F., Poupon, C., Poupon, F.: Real-time Rician noise correction applied to real-time HARDI and HYDI. In: Workshop CDMRI of MICCAI, pp. 2-13 (2010)

12. Aja-Fernández, S., Tristán-Vega, A., Alberola-López, C.: Noise estimation in singleand multiple-coil magnetic resonance data based on statistical models. MRI 27, 1397-1409 (2009)

13. Rajan, J., Poot, D., Juntu, J., Sijbers, J.: Noise measurement from magnitude MRI using local estimates of variance and skewness. Phys. Med. Biol. 55, N441-N449 (2010)

14. Collins, D., Zijdenbos, A., Kollokian, V., Sled, J., Kabani, N., Holmes, C., Evans, A.: Design and construction of a realistic digital brain phantom. IEEE Trans. Med. Imag. 17, 463-468 (1998)

15. Descoteaux, M., Angelino, E., Fitzgibbons, S., Deriche, R.: Regularized, Fast, and Robust Analytical Q-Ball Imaging. Magn. Reson. Med. 58, 497-510 (2007) 